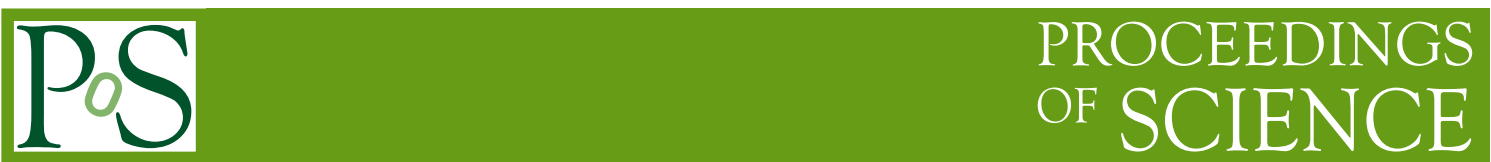

\title{
Inflationary observables and moduli masses
}

\author{
Kumar Das, Koushik Dutta* \\ Theory Division Saha Institute of Nuclear Physics, 1/AF Salt Lake, Kolkata - 700064, India \\ E-mail: kumar.dasesaha.ac.in, koushik.duttaesaha.ac.in
}

Anshuman Maharana

Harish Chandra Research Intitute, Chattnag Road, Jhunsi, Allahabad - 211019, India

E-mail: anshumanmaharana@hri.res.in

\begin{abstract}
The existence of moduli fields is generic in string/supergravity theories. The vacuum misalignment of moduli fields in inflationary and post-inflationary vacua usually leads to a matter dominated epoch in the post-inflationary history of the Universe. This modification in the evolutionary hierarchy of the Universe implies that the preferred range in the number of e-folds $N_{k}$ between the horizon exit of relevant modes in the CMB and the end of inflation is a function of moduli masses. As a result the CMB observables become sensitive to moduli masses. We have studied this sensitivity for some representative inflationary models using PLANCK 2015 data.
\end{abstract}

18th International Conference From the Planck Scale to the Electroweak Scale, 25-29 May 2015, Ioannina.

\footnotetext{
* Speaker.
} 


\section{Introduction}

Theory of inflation is the leading candidate in describing the early universe cosmology. Observationally it has received much support from the precision CMB measurements. The future experiments are geared towards pinning down the strength of tensor-to-scalar ratio with greater accuracy. Nevertheless, inflationary theory faces several challenges. The notable one is the sensitivity of the slow-roll conditions on ultra-violet degrees of freedom. Therefore, it requires considerations of inflation in a full theory of quantum gravity $[1,2,3]$, and string theory is one such framework to address this problem.

A generic feature of string theory is the existence of moduli fields which have Planck suppressed couplings to the low energy degrees of freedom. The minima of the modulus fields during inflation and after inflation are usually separated by a field range of order Planck mass. Due to this vacuum misalignment of moduli fields, there exists a possibility of non-standard post-inflationary cosmological timeline dominated by the oscillations of the cold moduli particles. After the decay of moduli fields, the history of the universe becomes thermal.

The aim of our work is to study the sensitivity of inflationary observables scalar spectral index $\left(n_{s}\right)$ and the tensor-to-scalar ratio $(r)$ to the mass of the lightest modulus for some familiar inflationary models [4]. The basic strategy is following: Given a model of inflation one can express $n_{s}$ and $r$ in terms of the number of e-folds $N_{k}$ between the horizon exit of the observational scales in the CMB and the end of inflation. A knowledge of $N_{k}$ then provides us the predictions of $n_{s}$ and $r$. In standard cosmological history of the Universe where inflation is followed by reheating producing radiation domination and subsequent matter domination, the preferred range for $N_{k}$ is $55 \pm 5$. The ambiguity of $\Delta N= \pm 5$ arises mainly due to our poor understanding about the details of the reheating epoch. Incorporation of the modulus dominated epoch at the end of reheating alters the preferred range to $N_{k}=\left(55-\frac{1}{4} N_{\text {mod }}\right) \pm 5$, where $N_{\text {mod }}$ being the e-folds of modulus domination that depends on the mass of the lightest modulus $m_{\varphi}[5,4]$. Therefore, the predictions for an inflationary model for $n_{s}$ and $r$ will be related to the mass of the lightest modulus through their dependence on $N_{k}$. Treating $m_{\varphi}$ as a free parameter and using PLANCK 2015 data [6], we will study this sensitivity for four representative models of inflation: quadratic potential [7], axion monodormy [8], natural inflation [9] and Starobinsky inflation [10]. The results turn out to be interesting as the predictions of the models change considerably when the modulus dominated epoch is taken care properly.

\section{Modular cosmology in brief}

Moduli are massless scalar fields that occur naturally in many string compactifications. Their interactions to other particles are suppressed by the Planck scale. The moduli usually have contributions to its masses during inflation that vanishes when inflation ends. Therefore the moduli get stuck at its local minima with its inflationary mass larger than the Hubble sale during inflation $H_{\text {inf }}$. For a modulus whose post-inflationary mass $m_{\varphi}$ is less than $H_{\text {inf }}$, the field starts to move to its global minima when the Hubble constant becomes smaller than $m_{\varphi}$ - leading to coherent oscillations of the moduli fields. Typically the initial amplitude of the oscillations are of the order of Planck mass $[11,12,13,14,15]$. At the end of inflation the universe reheats from the decays of 
inflaton, and the total energy density consists of two components: radiation from the reheating, and the oscillation energy of the displaced modulus. The energy density of the modulus oscillations decreases as a non-relativistic matter, and starts to dominate over the radiation density soon. The duration of this epoch is governed by the life time of the modulus $\tau_{m o d} \simeq 16 \pi M_{p l}^{2} / m_{\varphi}^{3}$. After the decay of the modulus the universe reheats once again, and there from the history of the Universe becomes thermal. In modular cosmology, we have the following evolutionary hierarchy: inflation, reheating (from inflaton decay), radiation domination, modulus domination, reheating (from modulus decay), matter domination, and finally accelerated expansion of today.

Now the total energy density $\rho_{k}$ when a cosmologically relevant perturbation of comoving wave number $k$ exits the horizon, must be evolved to the present day energy density $\rho_{0}$. The later is known from the CMB observations. Any theoretical proposal that one ascribes to the history of the universe must be consistent with the above condition. This criterion gives us the following consistency relation for the modular cosmology [5]

$$
N_{k} \approx\left(55.43-\frac{1}{4} N_{\text {mod }}\right)+\left(\frac{1}{4}\left(1-3 w_{r e 1}\right) N_{r e 1}+\frac{1}{4}\left(1-3 w_{r e 2}\right) N_{r e 2} \frac{1}{4} \ln r+\frac{1}{4} \ln \left(\rho_{k} / \rho_{\text {end }}\right)\right),
$$

where $N_{r e 1}, w_{r e 1}$ are the e-folds number and effective equation of state parameter of first reheating phase respectively, $N_{r e 2}, w_{r e 2}$ are the corresponding quantities for second reheating phase and $\rho_{\text {end }}$ is the energy density at end of inflation. Form Eq. (2.1), the preferred range $N_{k}$ turns out to be,

$$
N_{k}=\left(55-\frac{1}{4} N_{\text {mod }}\right) \pm 5, \quad \text { with } \quad N_{\text {mod }} \approx \frac{4}{3} \ln \left(\frac{\sqrt{16 \pi} M_{p l} Y^{2}}{m_{\varphi}}\right) .
$$

Here $Y$ is the initial displacement of the modulus in Planck unit ${ }^{1}$. Thus we see that the central value of $N_{k}$ is lowered by $\left(N_{\bmod } / 4\right)$, and it is determined by the modulus mass $m_{\varphi}$ and the initial displacement $Y$.

\section{Predictions for various inflationary models}

Here we will be studying the phenomenological implications of the existence of modulus dominated epoch on various inflationary models. We let $m_{\varphi}$ to be a phenomenological parameter between $10^{3} \mathrm{TeV}<m_{\varphi}<10^{8} \mathrm{TeV}$ and take $Y \sim 0.1 M_{p l}$. The lower bound for the mass is set by the cosmological moduli problem (CMP) bound i.e. $m_{\varphi}>30 \mathrm{TeV}$ for successfull nucleosynthesis [16, 17, 18]. The upper bound is set from the numerical solution of the following condition

$$
m_{\varphi}<H_{k}, \quad \text { where } \quad H_{k}=\frac{\pi}{\sqrt{2}}\left(r A_{s}\right)^{1 / 2} M_{p l},
$$

and it usually turns out to be $m_{\varphi} \sim 10^{10} \mathrm{TeV}$. Here $A_{s}$ is the amplitude of curvature perturbation. Since $r$ decreases with $N_{k}$, the aforesaid condition can be implemented over the entire range of $N_{k}$ if it holds for the maximum value $N_{\max }=60-\frac{1}{3} \ln \left(\sqrt{16 \pi} Y M_{p l} / m_{\varphi}\right)$.

With these considerations, we have finally evaluated the predictions for $n_{s}$ and $r$ for $m^{2} \chi^{2}$, axion monodormy, natural inflation and Starobinsky inflation taking $m_{\varphi}=10^{3}, 10^{6}$ and $10^{8} \mathrm{TeV}$. The resuts are shown in Fig.(1). The shaded regions are 1- $\sigma$ and $2-\sigma$ contours of PLANCK 2015

\footnotetext{
${ }^{1} Y$ can be explicitly computed in the full high energy theory that embeds inflation. We take $Y$ as a free parameter.
} 

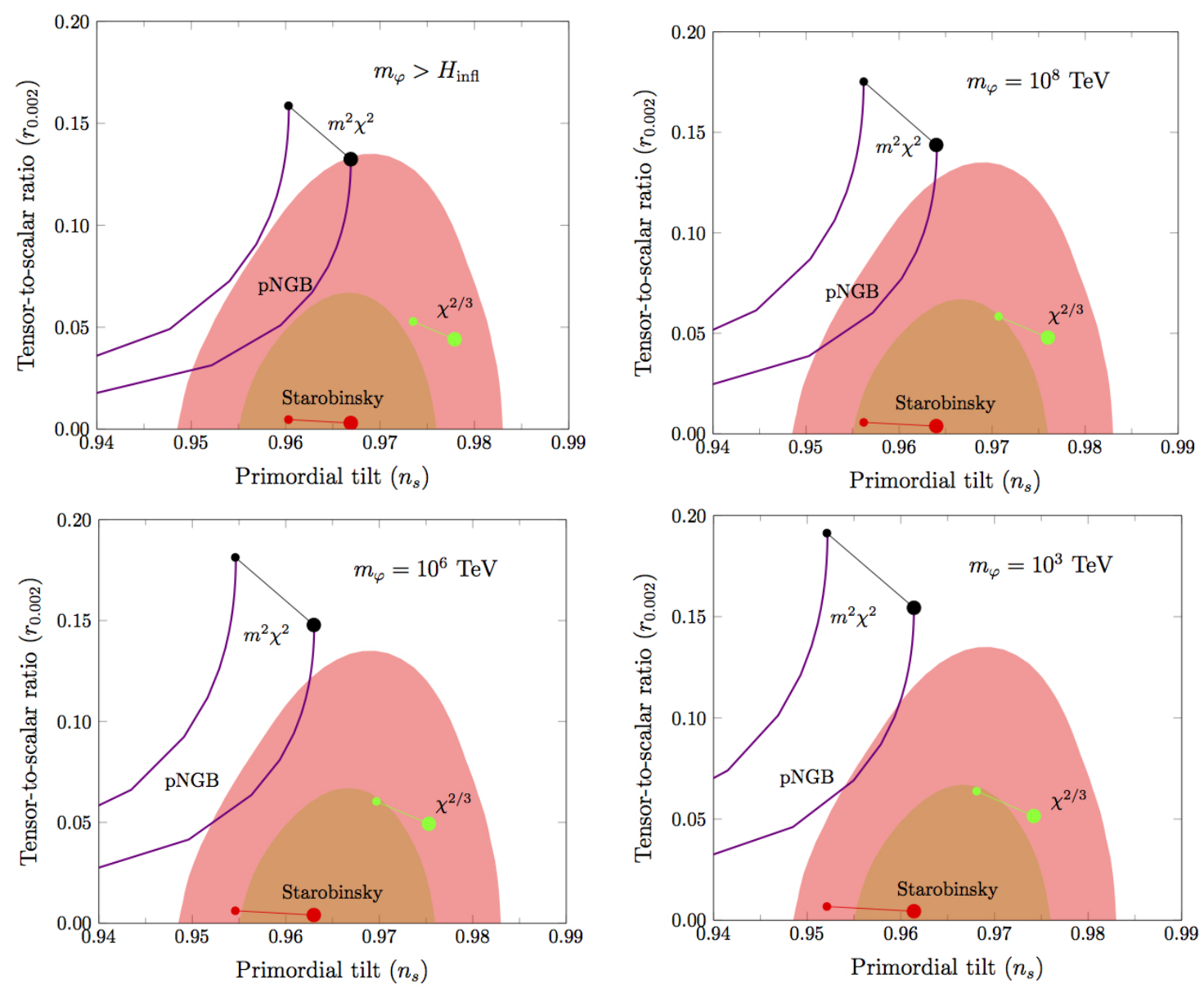

Figure 1: Inflationary predictions for $m^{2} \chi^{2}$ (black), Natural/pNGB inflation (purple), Axion monodromy (green), Starobinsky model (red) for cases of no misalignment $\left(m_{\varphi}>H_{\text {infl }}\right), m_{\varphi}=10^{3}, 10^{6}, 10^{8} \mathrm{TeV}$.

$\mathrm{TT}+$ low P [6]. We see that for the entire preferred range, the Starobinsky model lies within 1- $\sigma$ contour. However, for the heavy modulus $10^{8} \mathrm{TeV}$ the $m^{2} \chi^{2}$ is outside the 2- $\sigma$, while the axion monodromy enters in the $1-\sigma$ for $m_{\varphi}$ below $10^{5} \mathrm{TeV}$. For gravity mediated SUSY breaking models, our results correlate the inflationary predictions to the scale of SUSY breaking since $m_{S U S Y} \sim m_{\varphi}$.

\section{A bound on modulus mass}

Using the consistency relation of Eq. (2.1), and assuming non-exotic reheating phases, i.e. $w_{r e 1}, w_{r e 2}<1 / 3[19,20]$, we can get a lower bound on the modulus mass

$$
m_{\varphi} \gtrsim \sqrt{16 \pi} M_{p l} Y^{2} e^{-3\left(55.43-N_{k}+\frac{1}{4} \ln \left(\rho_{k} / \rho_{\text {end }}\right)+\frac{1}{4} \ln r\right)} .
$$

If the modulus mass is lower than the above bound, it decays too late in eating up larger number of e-folds that violates the consistency conditions. The above formula is applicable only for $m_{\varphi}<$ $H_{\text {inf }}$. Taking observational inputs of $n_{s}, N_{k}$ can be computed for a given inflationary model. With PLANCK 2015 central value of $n_{s}$ we found that the bound is incompatible for $m^{2} \chi^{2}$ model of 


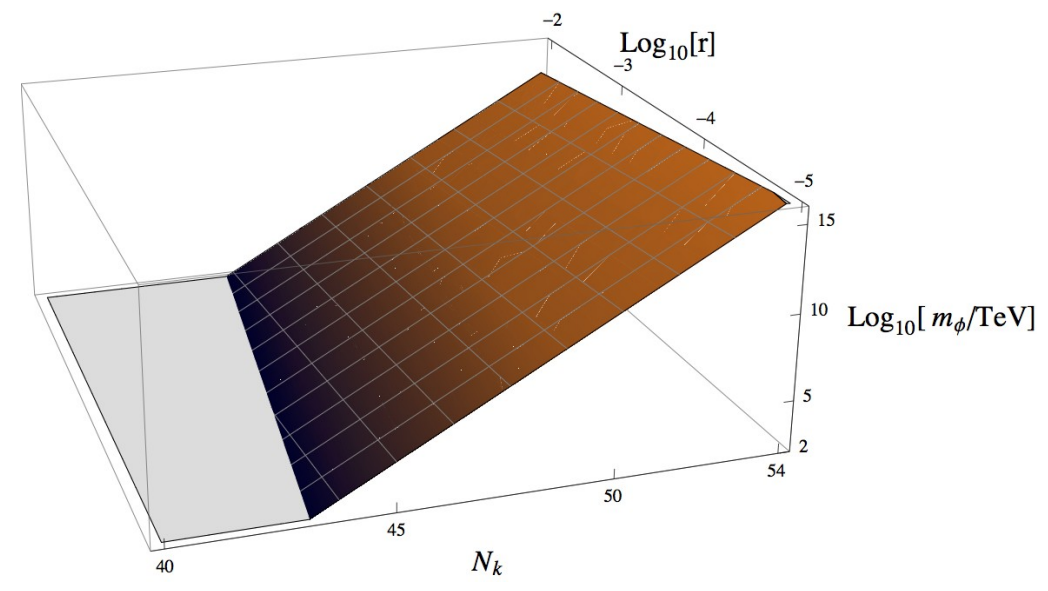

Figure 2: Bound on the modulus mass for small field models. The allowed values of $m_{\varphi}$ are in the region above the shaded plane.

inflation. For axion monodormy models the bound is far below the CMP bound. For small field models, Fig. (2) shows the allowed range in $m_{\varphi}$ with varitaions of $N_{k}$ and $r$. It shows that the bound is essentially determined by $N_{k}$. For $N_{k} \gtrsim 44.5$ the bound is stronger than CMP bound.

\section{Conclusion}

The motivation of our work is to study the sensitivity of inflationary observables with the mass of the lightest modulus where post-inflationary epoch is dominated by the coherent oscillations of the modulus field. We have kept the modulus mass as a free parameter and worked with a generic value for initial modulus displacement at the end of inflation. Based on this phenomenological approach the results will have broad implications. It suggests the importance of taking care of the modulus dominated epoch in computing the relevant e-folds $N_{k}$ of inflation. In future experiments [21], the uncertainty in measurement of $n_{s}$ is expected to reduce by an order of magnitude, and the dependence of modulus mass on observable predictions is crucial. In addition, this study also encourages us to address the issue of explicit computation of initial displacement of the modulus for specific models in a full UV theory.

\section{References}

[1] C. Burgess, M. Cicoli, and F. Quevedo arXiv:1306.3512 [hep-th]

[2] E. Silverstein, arXiv:1311.2312 [hep-th]

[3] D. Baumann and L. McAllister, arXiv:1404.2601 [hep-th].

[4] K. Das, K. Dutta and A. Maharana, arXiv:1506.05745 [hep-ph].

[5] K. Dutta and A. Maharana, Phys. Rev. D 91 (2015) 4, 043503 [arXiv:1409.7037 [hep-ph]].

[6] P. A. R. Ade et al. [Planck Collaboration], arXiv:1502.02114 [astro-ph.CO].

[7] A. D. Linde, Phys. Lett. B 129 (1983) 177. 
[8] L. McAllister, E. Silverstein and A. Westphal, Phys. Rev. D 82, 046003 (2010) [arXiv:0808.0706 [hep-th]]. E. Silverstein and A. Westphal, Phys. Rev. D 78, 106003 (2008) [arXiv:0803.3085 [hep-th]].

[9] F. C. Adams, J. R. Bond, K. Freese, J. A. Frieman and A. V. Olinto, Phys. Rev. D 47 (1993) 426 [hep-ph/9207245].

[10] A. A. Starobinsky, Phys. Lett. B 91 (1980) 99.

[11] M. Dine, W. Fischler and D. Nemeschansky, Phys. Lett. B 136 (1984) 169.

[12] G. D. Coughlan, R. Holman, P. Ramond and G. G. Ross, Phys. Lett. B 140 (1984) 44.

[13] M. Dine, L. Randall and S. D. Thomas, Phys. Rev. Lett. 75 (1995) 398 [hep-ph/9503303].

[14] M. Dine, L. Randall and S. D. Thomas, Nucl. Phys. B 458 (1996) 291 [hep-ph/9507453].

[15] A. S. Goncharov, A. D. Linde and M. I. Vysotsky, Phys. Lett. B 147 (1984) 279.

[16] M. Kawasaki, K. Kohri and N. Sugiyama, Phys. Rev. Lett. 82, 4168 (1999) [astro-ph/9811437].

[17] G. D. Coughlan, W. Fischler, E. W. Kolb, S. Raby and G. G. Ross, Phys. Lett. B 131 (1983) 59;

[18] B. de Carlos, J. A. Casas, F. Quevedo and E. Roulet, Phys. Lett. B 318 (1993) 447 [hep-ph/9308325].

[19] P. A. R. Ade et al. [Planck Collaboration], arXiv:1303.5082 [astro-ph.CO].

[20] L. Dai, M. Kamionkowski and J. Wang, Phys. Rev. Lett. 113 (2014) 041302 [arXiv:1404.6704 [astro-ph.CO]].

[21] L. Amendola et al. [Euclid Theory Working Group Collaboration], Living Rev. Rel. 16, 6 (2013) [arXiv:1206.1225 [astro-ph.CO]]. P. Andre et al. [PRISM Collaboration], arXiv:1306.2259 [astro-ph.CO]. A. Kosowsky, Physics 3, 103 (2010). 